\title{
Estimativas volumétricas em povoamentos de eucalipto sob regime de alto fuste e talhadia no sudoeste da Bahia
}

\author{
Gileno Brito de Azevedo¹, Glauce Taís de Oliveira Sousa¹, Patrícia Anjos Bittencourt Barreto¹, Valdemiro Conceição Júnior ${ }^{1}$
}

${ }_{1}^{1}$ Universidade Estadual do Sudoeste da Bahia (UESB), Estrada do Bem Querer, Km 4, CEP 45083-900, Vitória da Conquista, BA, Brasil

"Autor correspondente:

gilenoba@hotmail.com

Termos para indexação:

Eucalyptus urophylla

Modelos volumétricos

Fator de forma

Quociente de forma

Manejo florestal

Index terms:

Eucalyptus urophylla

Volumetric models

Form factor

Form quotient

Forest management

Histórico do artigo:

Recebido em 15 jun 2011

Aprovado em 07 dez 2011

Publicado em 28 dez 2011

doi: 10.4336/2011.pfb.31.68.309
Resumo - Esse estudo teve como objetivo testar modelos volumétricos e avaliar a eficiência de três métodos para estimar o volume de madeira em plantios de Eucalyptus urophylla, manejados sob regime de alto fuste e talhadia no Município de Vitória da Conquista, BA. Foi realizada cubagem rigorosa de árvores para obtenção do volume individual, e com os dados foram ajustados sete modelos volumétricos. Os melhores modelos foram selecionados com base no critério de seleção do valor ponderado dos escores dos parâmetros estatísticos (VP). Os volumes obtidos por meio dos três métodos (fator de forma, quociente de forma e modelos ajustados) foram comparados ao volume obtido na cubagem. O modelo de Schumacher e Hall logaritmizado e de Spurr foram os mais indicados para estimar o volume dos povoamentos nas duas condições de manejo. $\mathrm{O}$ volume obtido a partir dos dois melhores modelos ajustados resultou em estimativas mais próximas do volume real quando comparados aos volumes estimados pelo fator de forma e quociente de forma.

\section{Volumetric estimates in eucalypt plantation under regime of high forest and coppice in the southwest of Bahia, Brazil}

\begin{abstract}
This study aimed to test volumetric models and check the the efficiency of three methods to estimate the wood volume in Eucalyptus urophylla plantation, managed under regime of high forest and coppice, on a property located in the county of Vitória da Conquista, Bahia. It was carried out rigorous measurements scaled trees to obtain the volume to adjust seven volumetric models. The best models were selected using the score pondered value of the statistical parameters (VP). The volumes obtained by three methods (form factor, form quotient and adjusted models) were compared to the volume obtained in the scaling. The models of Schumacher e Hall $(\log )$ and Spurr were the more indicated to estimate the volume of stands in both management conditions. The volume obtained from the two best adjusted models resulted in estimates closer to the real volume when compared to the volumes estimated by form factor and the form quotient.
\end{abstract}

\section{Introdução}

O volume é uma das variáveis de maior importância para o diagnóstico do potencial produtivo de uma floresta, constituindo uma informação básica em qualquer processo de planejamento da produção florestal.
A literatura apresenta vários métodos que podem ser utilizados para a estimativa da produção volumétrica de uma floresta. De acordo com Soares et al. (2006), os mais comumente utilizados são o fator de forma, o quociente de forma e o ajuste de modelos volumétricos, gerados por meio da utilização de dados de cubagem 
rigorosa de árvores abatidas ou de árvores cubadas em pé. Guimarães \& Leite (1996) consideram o emprego de modelos volumétricos o procedimento mais eficiente para quantificação do volume de um povoamento florestal.

Muitas equações matemáticas foram desenvolvidas para estimar o volume de povoamentos florestais, sendo o modelo de Schumacher \& Hall (1933) o principal modelo utilizado por empresas do setor privado (Oliveira et al., 2009). No entanto, mesmo os modelos mais eficientes, nem sempre se ajustam a todas as espécies e condições, sendo recomendável testá-los por meio de estatísticas adequadas, identificando o mais indicado para cada situação (Thomas et al., 2006). A seleção de equações é uma fase fundamental na predição da produção, pois qualquer erro de tendência na estimativa do volume por árvore terá reflexos na estimativa da população, causando uma sub ou superestimação (Campos et al., 1985).

Existe um grande número de estudos analisando diferentes métodos de estimação do volume e equações volumétricas para o gênero Eucalyptus realizados no Brasil (Silva \& Araújo, 1984; Couto \& Bastos, 1987; McTague et al., 1989; Guimarães \& Leite, 1996; Franco et al., 1997; Schneider et al., 1997; Oliveira et al., 2009; Miguel et al., 2010; Soares et al., 2010; Azevedo et al., 2011), entretanto, poucos foram aqueles desenvolvidos no Estado da Bahia, de uma maneira geral (Silva et al., 1984; Rocha et al., 2010; Almeida et al., 2011), apesar deste apresentar a terceira maior área plantada com o gênero no Brasil (Anuário..., 2010). Dessa forma, estudos relacionados a esse tema na Bahia e, em particular, na região Sudoeste do Estado, onde, como consequência de uma elevada demanda energética vegetal, percebe-se uma grande ascensão das áreas plantadas com eucalipto nos últimos anos, podem ser de grande importância para a economia e fortalecimento do setor florestal da região.

Nesse contexto, o presente trabalho teve como objetivos: (a) testar diferentes modelos volumétricos e selecionar as equações de melhor ajuste e precisão e (b) avaliar a eficiência de três diferentes métodos para estimar o volume de madeira (fator de forma, quociente de forma e emprego equações volumétricas ajustadas) para a espécie Eucalyptus urophylla sob dois regimes de manejo (alto fuste e talhadia) no Município de Vitória da Conquista, BA.

\section{Material e métodos}

\section{Área de estudo}

Os dados utilizados foram obtidos em dois povoamentos de Eucalyptus urophylla, com cerca de 5 anos de idade, conduzidos sob dois regimes de manejo (alto fuste e talhadia), localizados na Fazenda Bela Vista, situada no Distrito de Pradoso, pertencente ao Município de Vitória da Conquista, Bahia. A escolha por trabalhar com plantações com cerca de 5 anos de idade foi baseada no fato de que a grande maioria das plantações florestais da região são destinadas à produção de madeira para fins energéticos, com ciclos de corte entre 4 e 5 anos de idade.

A região tem clima classificado como tropical de altitude (Cwb), segundo a classificação de Köppen, e relevo plano a levemente ondulado, com altitude em torno de $880 \mathrm{~m}$. A temperatura média anual é de $21^{\circ} \mathrm{C}$ e a precipitação varia entre $700 \mathrm{~mm}$ e $1.100 \mathrm{~mm}$ anuais, distribuída nos meses de novembro a março, com um período seco de quatro a cinco meses. A vegetação é classificada como Floresta Estacional Semidecidual Montana, também denominada de "Mata de Cipó". $\mathrm{O}$ solo da região pertence à classe Latossolo Amarelo Distrófico (Novaes et al., 2008).

\section{Coleta de dados}

Foram selecionadas e abatidas 35 árvores no povoamento sob regime alto fuste, distribuídas em quatro classes diamétricas com amplitude de $2 \mathrm{~cm}$ entre classes, sendo observada uma variação de DAP (diâmetro a altura do peito) de $8,69 \mathrm{~cm}$ a $16,87 \mathrm{~cm}$ e em altura de $10,2 \mathrm{~m}$ a $16,5 \mathrm{~m}$. Já no povoamento sob regime de talhadia, foram abatidas 59 árvores, originadas de 30 cepas selecionadas aleatoriamente, com DAP variando de $6,21 \mathrm{~cm}$ a $15,25 \mathrm{~cm}$ e altura de $10,6 \mathrm{~m}$ a $16,84 \mathrm{~m}$. Nesse último povoamento, para efeito de cálculos e ajuste de equações, cada fuste resultante da condução dos brotos foi considerado uma árvore.

\section{Cubagem rigorosa}

A cubagem das árvores foi realizada através do método de Smalian. No povoamento em alto fuste, as circunferências com casca ao longo do fuste foram medidas nas posições de $0 \mathrm{~m}, 0,3 \mathrm{~m}, 0,7 \mathrm{~m}, 1,3 \mathrm{~m}$ e $2 \mathrm{~m}$ do nível do solo e a partir desse ponto as seções foram medidas de 2 em $2 \mathrm{~m}$ até a altura onde se encontrava diâmetro com casca de cerca de $4 \mathrm{~cm}$. A partir dessa 
última posição, foi medido também o comprimento da ponta. No povoamento sob regime talhadia, os procedimentos foram os mesmos, exceto para a medida da circunferência inicial que foi obtida na inserção de cada fuste sobre a cepa. A escolha do diâmetro mínimo comercial de $4 \mathrm{~cm}$ foi baseada no objetivo da produção que será destinada a fins energéticos.

As circunferências das seções e as alturas das árvores abatidas foram medidas com auxílio de fita métrica e trena, respectivamente. Após a transformação das circunferências para diâmetros, foram calculados os volumes por seção e, em seguida, o volume total de cada árvore foi obtido pelo somatório do volume de todas as seções, incluindo o volume da ponta, e o volume comercial foi obtido pelo somatório das seções até a última seção, delimitada pelo diâmetro mínimo considerado.

\section{Métodos para predição do volume}

Foram adotados três métodos usualmente utilizados para estimativa do volume de madeira: (a) fator de forma, (b) quociente de forma e (c) ajuste de modelos volumétricos.

O fator de forma de cada árvore foi obtido pela razão entre o volume real, adquirido a partir do método de Smalian, e o volume do cilindro com diâmetro na altura de 1,30 m (Soares et al., 2006). A partir da média aritmética desses valores individuais, obteve-se também o fator de forma médio de cada povoamento, o qual foi multiplicado pelo volume cilíndrico de cada árvore para obtenção do volume individual.

O quociente de forma representa o decréscimo natural ao longo do fuste e é definido pela razão entre diâmetros. Foi utilizado o quociente de forma de Schiffel que é dado

por: $Q=\frac{D_{1 / 2 H}}{D A P}$

sendo $Q<1$, onde $D_{1 / 2 H}=$ diâmetro medido na metade da altura total da árvore. Da mesma maneira que para o fator de forma, o volume de cada árvore foi obtido pela multiplicação do quociente de forma médio do povoamento pelo volume do cilindro.

$\mathrm{O}$ ajuste de modelos volumétricos foi utilizado para a predição do volume total e do volume comercial dos povoamentos. Os modelos adotados apresentam como variável dependente o volume $(\mathrm{V})$ em função das variáveis independentes altura total $(\mathrm{Ht})$ e/ou do diâmetro das árvores, ao nível do solo (DNS) ou à altura do peito (DAP). Foram avaliados sete modelos, seis encontrados na literatura e um proposto no presente trabalho (Tabela 1), a fim de identificar quais os mais adequados a cada regime de manejo. O modelo proposto apresenta como característica a utilização das variáveis independentes DAP e DNS, em vez da variável $\mathrm{H}$. Por ser de fácil obtenção, o DNS pode ser uma alternativa em situações onde não se pode obter a variável altura ou quando se deseja otimizar o tempo de medição em pequenas áreas plantadas.

Tabela 1. Modelos de relação volumétrica utilizados para estimar o volume dos indivíduos dos povoamentos.

\begin{tabular}{cll}
\hline $\mathbf{N}^{\mathbf{o}}$ & \multicolumn{1}{c}{ Modelo } & \multicolumn{1}{c}{ Autor } \\
\hline $\mathbf{1}$ & $V=\beta_{0}+\beta_{1} \cdot D A P+\varepsilon$ & $\begin{array}{l}\text { Equação da reta } \\
\text { Koperzky e } \\
\text { Gehrhardt }\end{array}$ \\
$\mathbf{2}$ & $V=\beta_{0}+\beta_{1} \cdot D A P^{2}+\varepsilon$ & $\begin{array}{l}\text { Hohenald e } \\
\text { Krenm }\end{array}$ \\
$\mathbf{3}$ & $V=\beta_{0}+\beta_{1} \cdot D A P+\beta_{2} . D A P^{2}+\varepsilon$ & Proposto \\
$\mathbf{4}$ & $V=\beta_{0}+\beta_{1} \cdot D A P^{2}+\beta_{2} \cdot D N S^{2} \varepsilon$ & Schumacher e \\
$\mathbf{5}$ & $V=\beta_{0}+\beta_{1} \cdot \operatorname{Ln}(D A P)+\beta_{2} \cdot \operatorname{Ln}(H)+\varepsilon$ & Hall modificado \\
& & $\begin{array}{l}\text { Schumacher e Hall } \\
\text { (logaritimizado) }\end{array}$ \\
$\mathbf{6}$ & $\operatorname{Ln}(V)=\beta_{0}+\beta_{1} \cdot \operatorname{Ln}(D A P)+\beta_{2} \cdot \operatorname{Ln}(H)+\varepsilon$ \\
7 & $\operatorname{Ln}(V)=\beta_{0}+\beta_{1} \cdot \operatorname{Ln}\left(D A P^{2} \cdot H\right)+\varepsilon$ & Spurr \\
\hline
\end{tabular}

Em que: $\beta_{\mathrm{i}}=$ coeficientes dos modelos; $\mathrm{H}=$ altura total $(\mathrm{m}) ; \mathrm{DAP}=$ diâmetro com casca a altura do peito $(\mathrm{cm})$; DNS = diâmetro com casca ao nível do solo $(\mathrm{cm}) ; \mathrm{Ln}=$ Logaritmo neperiano; e $\varepsilon=$ erro aleatório.

\section{Critérios para seleção dos modelos matemáticos}

A seleção do melhor modelo de regressão foi baseada nos seguintes critérios: (a) Coeficiente de determinação ajustado em porcentagem $\left(\mathrm{R}^{2}{ }_{\mathrm{aj}} \%\right)$, (b) Erro padrão da estimativa $\left(\mathrm{S}_{\mathrm{yx}}\right)$, (c) Coeficiente de variação $(\mathrm{CV} \%)$, (d) Valor de F; e (e) Valor ponderado dos escores Estatísticos (VP). Também foi realizada a análise gráfica de resíduos para verificar a presença de tendenciosidade nas estimativas do volume.

(a) $\mathrm{O}$ coeficiente de determinação $\left(\mathrm{R}^{2}\right)$ informa a porcentagem da variação dos dados observados em torno da média que está sendo explicada pelo modelo ajustado. Os valores de $\mathrm{R}^{2}$ foram transformados (Scolforo, 1993), para que fosse possível comparar os valores encontrados, já que os graus de liberdade da regressão diferem entre os modelos testados. A expressão matemática utilizada para sua obtenção é dada por: 


$$
R_{a j}^{2}=1-\left(\frac{n-1}{n-p}\right) *\left(\frac{\text { Sq.res }}{S q \cdot t o t}\right)
$$

onde: $R_{a j}^{2}=$ coeficiente de determinação ajustado; $n=$ número de dados observados; $p=$ número de coeficientes do modelo; Sq.res = soma dos quadrados do resíduo para a variável de interesse; $S q$.tot $=$ soma dos quadrados total para a variável de interesse.

(b) $\mathrm{O}$ erro padrão da estimativa indica a precisão do ajuste de um modelo matemático. Esse critério informa o quanto, relativamente, o modelo erra em média ao estimar a variável dependente (Machado et al., 2008). É expresso por:

$$
S_{y x}=\sqrt{Q M_{r e s}}
$$

onde: $\mathrm{S}_{\mathrm{yx}}=$ erro padrão da estimativa; $Q M_{\text {res }}=$ quadrado médio do resíduo, obtido na análise de variância. Para os modelos logarítmicos o erro padrão da estimativa foi corrigido na escala original da variável dependente, para possibilitar a comparação com os modelos aritméticos. Essa correção foi feita com o índice de Furnival (Furnival, 1961).

(c) Quanto menor for o coeficiente de variação, melhor e mais precisa é a estimativa. O coeficiente é

obtido por: $C V=\frac{S_{y x}}{\bar{y}} * 100$

onde: $\mathrm{CV}=$ coeficiente de variação; $S_{y x}=$ erro padrão da estimativa; $\bar{y}=$ média aritmética da variável dependente.

(d) De acordo com o critério do valor de F, quanto maior o valor melhor será o ajuste do modelo (Thomas et al., 2006).

(e) O valor ponderado dos escores dos parâmetros estatísticos, segundo Thomas et al. (2006), leva em consideração todas as variáveis estatísticas consideradas como critérios e descritas anteriormente, sintetizando os resultados e facilitando o processo de seleção do melhor modelo matemático. $\mathrm{O}$ valor ponderado é determinado atribuindo-se pesos às variáveis estatísticas que foram, então, ordenadas de acordo com a sua eficiência, sendo atribuído peso 1 para o modelo mais eficiente, 2 para o segundo e assim sucessivamente, de acordo com a metodologia proposta por Thiersch (1997). Após a classificação individual, a pontuação de cada modelo foi somada, sendo selecionados os melhores modelos.

Os modelos selecionados através dos critérios considerados foram analisados quanto à distribuição gráfica dos resíduos, com propósito de verificar tendências na estimativa da variável dependente volume.

\section{Seleção dos métodos para obtenção do volume}

A fim de verificar qual método apresentou maior eficiência na estimativa do volume total de cada árvore, tendo como referência o valor obtido pela cubagem rigorosa, foram comparados os resultados de volume total encontrados por meio do fator de forma, do quociente de forma e dos modelos volumétricos selecionados em função do melhor desempenho.

Os resultados de volume obtidos para os dois regimes estudados foram analisados separadamente segundo um delineamento inteiramente casualizado com cinco tratamentos (cubagem rigorosa, fator de forma, quociente de forma, modelo Schumacher e Hall logaritimizado e o modelo de Spurr) com 7 e 11 repetições para os regimes de alto fuste e de talhadia, respectivamente. Adotou-se o teste F a 5\%. De forma complementar, para comparação de médias, utilizou-se o teste de Duncan a 5\% de significância. As análises foram realizadas empregando-se o programa SASMAgri desenvolvido por Canteri et al. (2001).

\section{Resultados e discussão}

\section{Ajuste dos modelos volumétricos}

Os valores dos coeficientes e parâmetros estatísticos provenientes do ajuste dos sete modelos volumétricos testados para a estimativa das variáveis volume total $(\mathrm{Vt})$ e volume comercial (Vc), em função das variáveis independentes (DAP, Ht e DNS), para os povoamentos de E. urophylla conduzidos pelo regime de alto fuste e de talhadia, estão apresentados nas Tabelas 2 e 3, respectivamente. 
Tabela 2. Coeficientes e parâmetros estatísticos obtidos no ajuste dos modelos volumétricos para povoamento de E. urophylla manejado sob regime de alto fuste, com 5 anos de idade, no Município de Vitória da Conquista, BA.

\begin{tabular}{|c|c|c|c|c|c|c|c|c|c|}
\hline & Modelo & $\boldsymbol{\beta}_{0}^{(l)}$ & $\beta_{1}$ & $\boldsymbol{\beta}_{2}$ & $\mathbf{R}_{\text {aj }}^{2}$ & $S_{y \cdot x}$ & CV \% & $\mathbf{F}$ & VP \\
\hline \multirow{7}{*}{ VT } & 1 & $-0,109408$ & 0,015228 & - & 90,04 & 0,0100 & 9,49 & 308,34 & 26 \\
\hline & 2 & $-0,012911$ & 0,000585 & - & 91,12 & 0,0091 & 8,96 & 349,90 & 18 \\
\hline & 3 & 0,014145 & 0,004222 & 0,000746 & 90,90 & 0,0090 & 8,94 & 352,21 & 17 \\
\hline & 4 & $-0,010661$ & 0,000630 & 0,000003 & 91,01 & 0,0092 & 9,02 & 173,01 & 24 \\
\hline & 5 & $-0,491076$ & 0,150559 & 0,076428 & 92,25 & 0,0085 & 8,37 & 203,23 & 15 \\
\hline & 6 & $-9,350878$ & 1,880630 & 0,802925 & 98,10 & 0,0047 & 4,66 & 880,48 & 4 \\
\hline & 7 & $-9,438418$ & 0,905947 & - & 98,09 & 0,0048 & 4,75 & 847,25 & 8 \\
\hline \multirow{7}{*}{$\mathrm{VC}$} & 1 & $-0,110483$ & 0,015238 & - & 90,01 & 0,0097 & 9,60 & 307,40 & 25 \\
\hline & 2 & $-0,013927$ & 0,000586 & - & 91,09 & 0,0092 & 9,07 & 348,77 & 18 \\
\hline & 3 & 0,013245 & $-0,004240$ & 0,000747 & 90,87 & 0,0091 & 8,96 & 351,07 & 17 \\
\hline & 4 & $-0,011715$ & 0,000630 & 0,000003 & 90,97 & 0,0092 & 9,13 & 172,32 & 23 \\
\hline & 5 & $-0,492350$ & 0,150669 & 0,076446 & 92,21 & 0,0086 & 8,48 & 202,26 & 15 \\
\hline & 6 & $-9,454351$ & 1,908742 & 0,810313 & 98,08 & 0,0048 & 4,74 & 871,62 & 4 \\
\hline & 7 & $-9,546139$ & 0,918335 & - & 98,07 & 0,0049 & 4,83 & 836,85 & 8 \\
\hline
\end{tabular}

${ }^{11} \beta_{\mathrm{i}}=$ coeficientes dos modelos; $\mathrm{R}^{2}$ = coeficiente de determinação ajustado; $\mathrm{S}_{\mathrm{yx}}=$ erro-padrão da estimativa; $\mathrm{CV}=$ coeficiente de variação; $\mathrm{F}=$ valor de $\mathrm{F}$ da análise de variância; $\mathrm{VP}=$ valor ponderado dos escores; $\mathrm{VT}=$ volume total; $\mathrm{VC}=$ volume comercial.

Tabela 3. Coeficientes e parâmetros estatísticos obtidos no ajuste dos modelos volumétricos para povoamento de E. urophylla manejado sob regime de talhadia, com 5 anos de idade, no Município de Vitória da Conquista, BA.

\begin{tabular}{|c|c|c|c|c|c|c|c|c|c|}
\hline & Modelo & $\boldsymbol{\beta}_{0}{ }^{(l)}$ & $\beta_{1}$ & $\boldsymbol{\beta}_{2}$ & $\mathbf{R}_{a j}^{2}$ & $\mathbf{S}_{\mathbf{y} . \mathbf{x}}$ & $\mathrm{CV} \%$ & $\mathbf{F}$ & VP \\
\hline \multirow{7}{*}{ VT } & 1 & $-0,080446$ & 0,013606 & - & 91,68 & 0,0097 & 13,26 & 648,76 & 22 \\
\hline & 2 & $-0,008614$ & 0,000615 & - & 93,35 & 0,0087 & 11,85 & 826,27 & 17 \\
\hline & 3 & 0,005884 & $-0,002700$ & 0,000735 & 93,19 & 0,0087 & 8,49 & 832,49 & 15 \\
\hline & 4 & $-0,010664$ & 0,000548 & 0,000004 & 93,47 & 0,0086 & 11,67 & 427,29 & 16 \\
\hline & 5 & $-0,424308$ & 0,107404 & 0,092440 & 90,99 & 0,0100 & 13,70 & 302,30 & 27 \\
\hline & 6 & $-10,279519$ & 1,843441 & 1,202448 & 98,01 & 0,0047 & 6,46 & 1473,19 & 4 \\
\hline & 7 & $-9,905314$ & 0,969562 & - & 97,97 & 0,0049 & 6,64 & 1395,69 & 8 \\
\hline \multirow{7}{*}{ VC } & 1 & $-0,082246$ & 0,013669 & - & 91,71 & 0,0097 & 13,49 & 651,71 & 22 \\
\hline & 2 & $-0,010061$ & 0,000617 & - & 93,34 & 0,0087 & 12,10 & 824,49 & 17 \\
\hline & 3 & 0,003015 & $-0,002435$ & 0,000726 & 93,17 & 0,0087 & 8,55 & 829,48 & 15 \\
\hline & 4 & $-0,012160$ & 0,000549 & 0,000005 & 93,46 & 0,0086 & 11,90 & 427,23 & 16 \\
\hline & 5 & $-0,427455$ & 0,108014 & 0,092669 & 91,06 & 0,0101 & 13,92 & 304,90 & 27 \\
\hline & 6 & $-10,517028$ & 1,908591 & 1,225368 & 97,88 & 0,0050 & 6,86 & 1374,53 & 4 \\
\hline & 7 & $-10,155692$ & 1,000491 & - & 97,85 & 0,0051 & 7,01 & 1314,80 & 8 \\
\hline
\end{tabular}

(1) $\beta_{\mathrm{i}}=$ coeficientes dos modelos; $\mathrm{R}_{\mathrm{aj}}^{2}=$ coeficiente de determinação ajustado; $\mathrm{S}$ = erro-padrão da estimativa; $\mathrm{CV}=$ coeficiente de variação; $\mathrm{F}=$ valor de $\mathrm{F}$ da análise de variância; $\mathrm{VP}=$ valor ponderado dos escores; $\mathrm{VT}=$ volume total; $\mathrm{VC}=$ volume comercial.

Os modelos testados apresentaram bom desempenho estatístico quanto aos valores de $\mathrm{R}_{\text {aj, }}^{2}$ que variou de $90,01 \%$ a $98,10 \%$, demonstrando, assim, alto grau de ajuste da variável dependente em função das variáveis independentes. Os modelos 6 e 7 apresentaram os maiores valores para essa estatística, em torno de
98\% nas duas situações estudadas. $\mathrm{O} \mathrm{S}_{\mathrm{yx}}$ também não apresentou grande variação entre os modelos estudados, estando entre $0,0047 \mathrm{~m}^{3}$ a $0,0101 \mathrm{~m}^{3}$.

Os coeficientes de variação $(\mathrm{CV} \%)$ dos modelos empregados foram menores no povoamento conduzido sob regime de alto fuste, com resultados variando entre 
$4,7 \%$ e $9,6 \%$. No povoamento sob regime de talhadia, apenas os modelos 3, 6 e 7 apresentaram valores inferiores a $10 \%$. Os valores de F calculados na análise de variância, em ambos os regimes de manejo estudados, foram mais significativos para os modelos 6 e 7 e menos significativos para os modelos 4 e 5 , indicando o melhor ajuste dos dois primeiros modelos.

De acordo com o valor ponderado (VP) (Tabelas $2 \mathrm{e}$ 3), os modelos 6 e 7 (Shumacher e Hall logaritmizado e Spurr, respectivamente) também foram os que apresentaram melhores resultados em todas as situações estudadas, sendo que o primeiro apresentou estatísticas de ajuste ligeiramente mais favoráveis do que o segundo. Este resultado corrobora com os de Rocha et al. (2010), que estimando o volume de E. urophylla no Planalto da Conquista na Bahia, através de diferentes modelos, encontraram melhor desempenho no modelo de Schumacher e Hall logaritmizado, com $\mathrm{R}^{2}$ igual a $95,64 \%$ e $\mathrm{S}_{\mathrm{yx}}$ de $0,0151 \mathrm{~m}^{3}$. Da mesma forma, Azevedo et al. (2011) também verificaram que esse modelo foi o mais indicado para estimar o volume com casca e sem casca de Eucalyptus sp. em Sergipe. Resultados semelhantes aos desses autores também foram encontrados por Tonini et al. (2009) ao estimar o volume com casca de Tectona grandis em Roraima.

Thomas et al. (2006) identificaram o modelo de Spurr entre os que apresentaram bons resultados na estimativa do volume com casca de Pinus taeda, com R ${ }_{\text {aj }}$ igual a 99,22\% e CV de -23,17\%. Para Moraes Neto (2009), esse mesmo modelo também apresentou bons ajustes para a estimativa do volume total com casca e sem casca de Pinus tecunumanii no Cerrado do Distrito Federal, com $\mathrm{R}_{\text {aj }}^{2}$ superior a $87 \%$.
O modelo 4, proposto no presente trabalho, apresentou valores de $\mathrm{R}^{2}{ }_{\text {aj }}$ superiores a $90 \%$ e CV inferiores a $12 \%$, no entanto, de acordo o valor do $\mathrm{VP}$, não esteve entre os melhores modelos em nenhum dos casos estudados. Dentre os modelos que estimam o volume apenas em função do DAP, o modelo de Hohenald e Krenm foi o que apresentou os menores valores de VP (17 para o regime de alto fuste e 15 para o regime de talhadia). Como a determinação da altura das árvores é uma operação onerosa e sujeita a erros (Couto \& Bastos, 1987), a avaliação da precisão desse tipo de modelo é relevante, pois pode representar redução de tempo e custos das operações de inventário florestal.

As Figuras 1 e 2 apresentam a distribuição gráfica dos resíduos em função do volume estimado (total e comercial) para os dois modelos selecionados de acordo com o VP. Nas mesmas figuras também estão apresentadas as distribuições dos resíduos observadas para o modelo de Hohenald e Krenn, selecionado entre os modelos que utilizam apenas o DAP como variável independente.

A distribuição gráfica dos resíduos não demonstrou tendências em nenhum dos três modelos selecionados, todavia, também indicou o melhor desempenho dos modelos de Schumacher e Hall logaritmizado e de Spurr quando comparados ao modelo de Hohenald e Krenm, que apresentou uma maior dispersão dos resíduos em relação a média, especialmente no regime de talhadia. Isso evidencia que esses dois modelos são os mais indicados para estimar o volume total e comercial da espécie nos dois regimes de manejo estudados. 

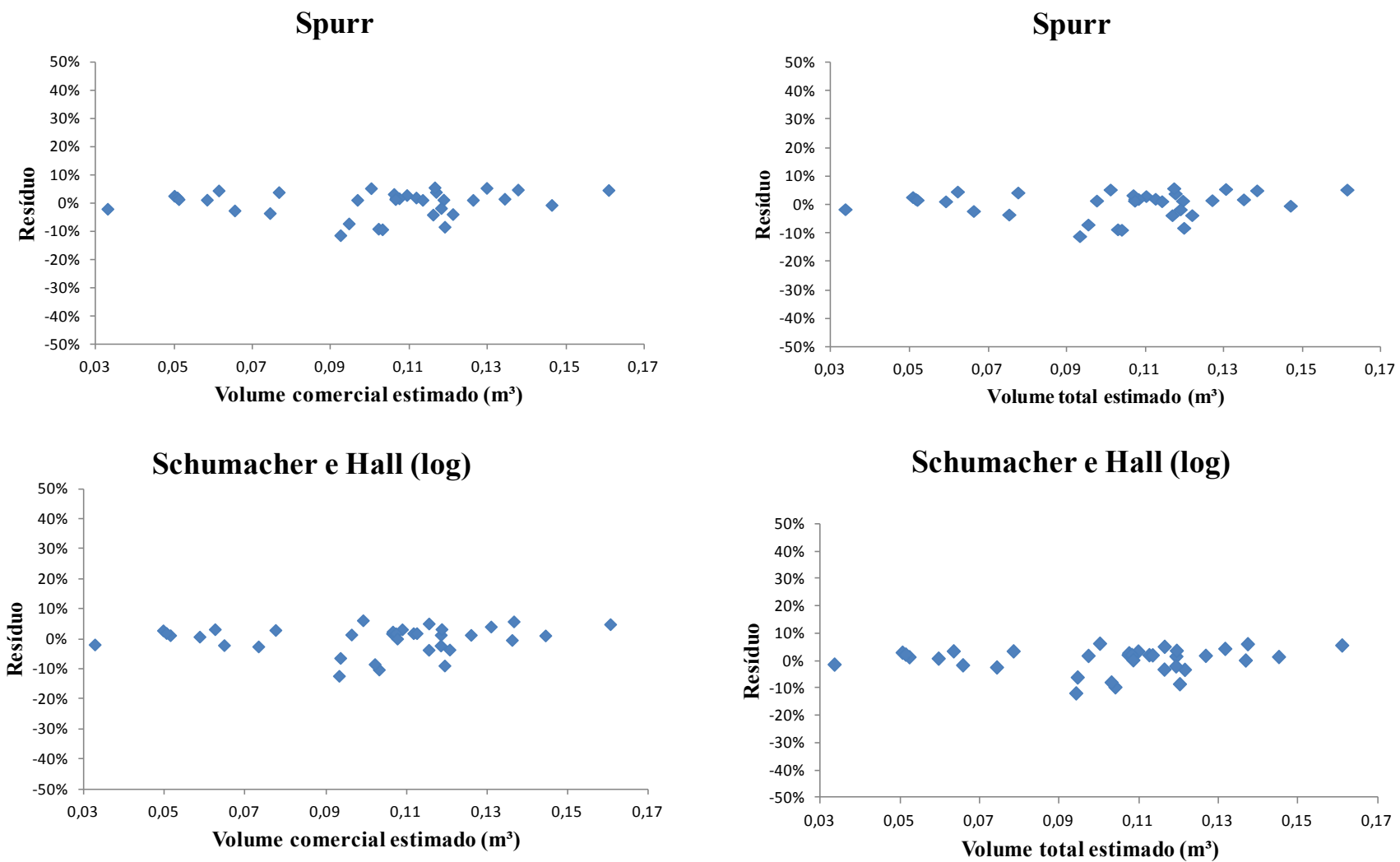

Hohenald e Krenm
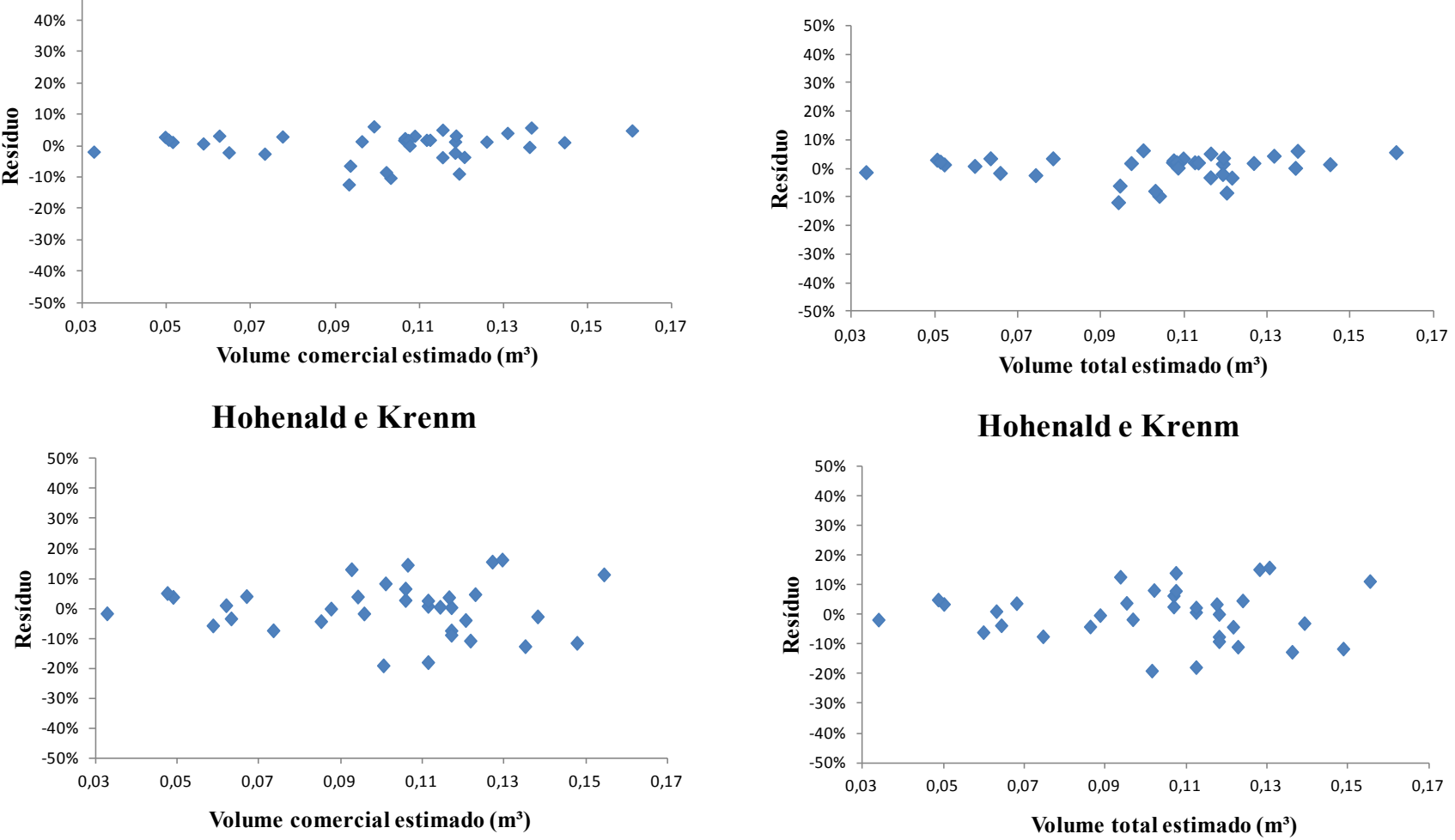

Hohenald e Krenm

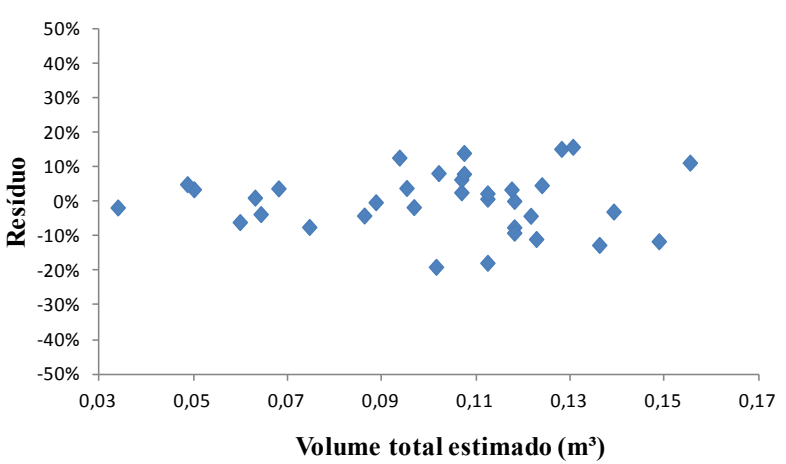

Figura 1. Distribuições dos resíduos em função do volume total estimado e do volume comercial estimado para os modelos selecionados para plantios de E. urophylla, com 5 anos de idade, manejados sob regime de alto fuste no Município de Vitória da Conquista, BA. 


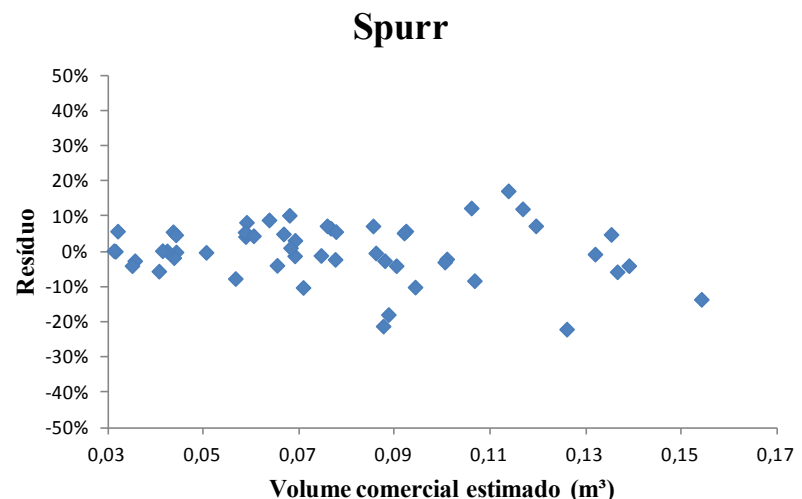

Schumacher e Hall (log)
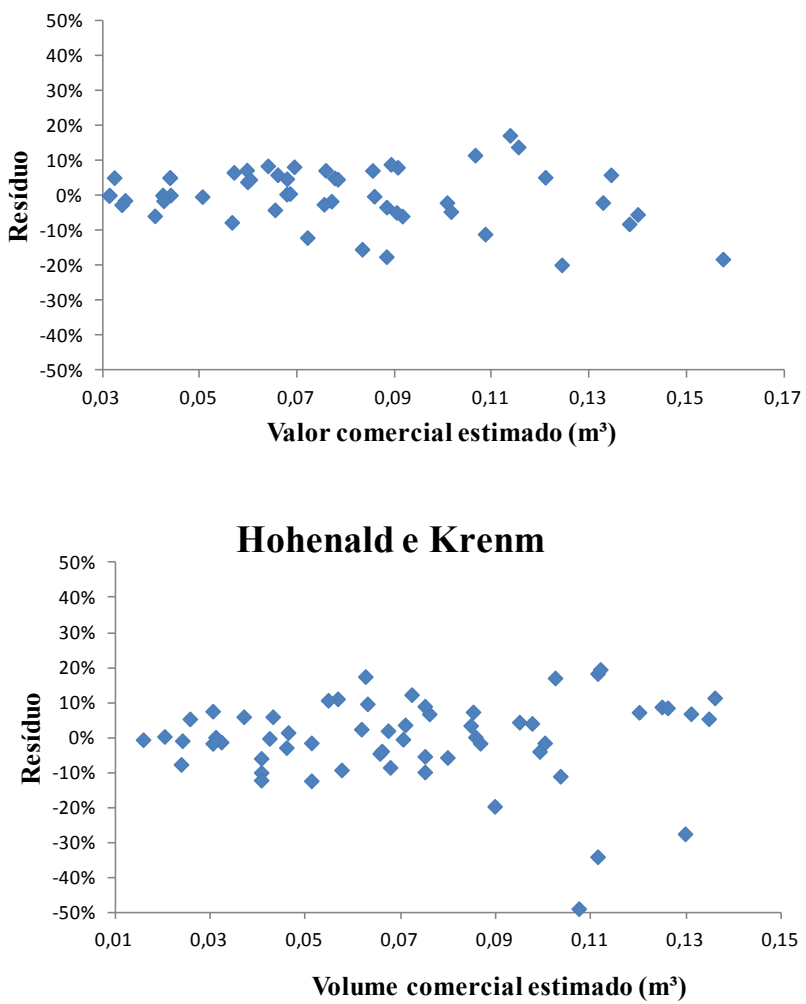

Spurr

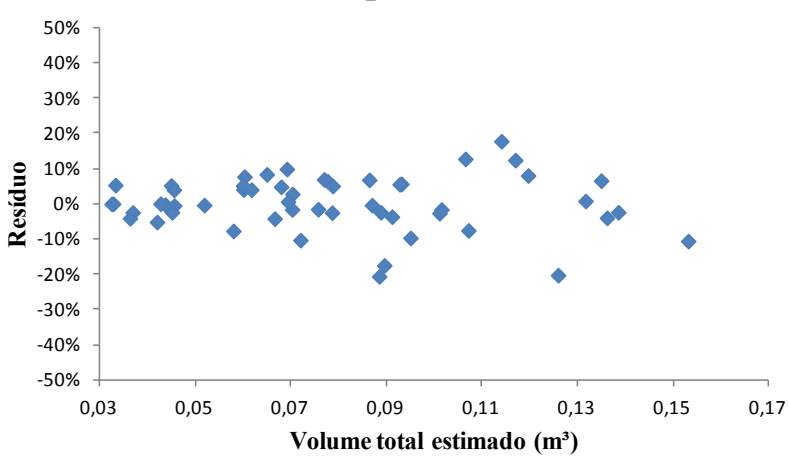

Schumacher e Hall (log)

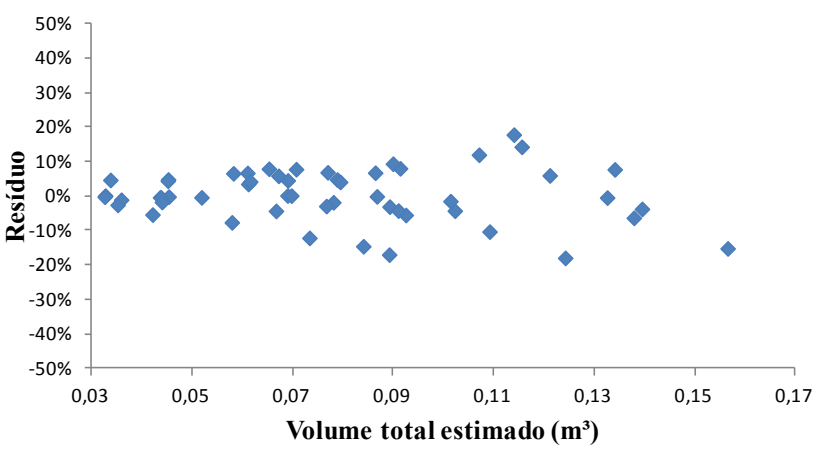

Hohenald e Krenm

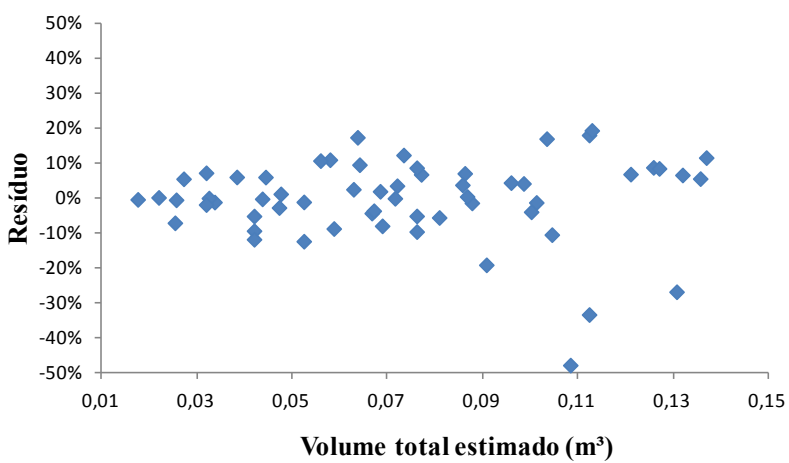

Figura 2. Distribuições dos resíduos em função do volume total estimado e do volume comercial estimado para os modelos selecionados para plantios de E. urophylla, com 5 anos de idade, manejados sob regime de talhadia no Município de Vitória da Conquista, BA.

\section{Comparação de métodos para estimativa do volume total}

A Tabela 4 apresenta os valores médios do volume real (cubagem rigorosa) e dos volumes estimados através do fator de forma médio, quociente de forma médio e pelos modelos ajustados de Schumacher e Hall logaritmizado e de Spurr. Os fatores e quocientes de forma médios foram, respectivamente: 0,49 e 0,64 (para o regime de alto fuste) e 0,51e 0,67 (para o regime de talhadia). 
Tabela 4. Resultados na análise estatística obtida pelos diferentes métodos de estimativa de volume individual de árvores de E. urophylla manejadas pelo regime de alto fuste e talhadia, no Município de Vitória da Conquista, BA.

\begin{tabular}{lccccc}
\hline \multicolumn{1}{c}{ Regime } & $\mathbf{V}(\mathbf{C R})^{(1)}$ & $\mathbf{V}(\boldsymbol{f})$ & $\mathbf{V}(\boldsymbol{Q})$ & $\mathbf{V}($ Mod. 1) & $\mathbf{V}($ Mod. 2) \\
\hline Alto fuste & $0,10192 \mathrm{~b}^{(2)}$ & $0,10393 \mathrm{~b}$ & $0,13575 \mathrm{a}$ & $0,10177 \mathrm{~b}$ & $0,10083 \mathrm{~b}$ \\
Talhadia & $0,07782 \mathrm{~b}$ & $0,07868 \mathrm{~b}$ & $0,10336 \mathrm{a}$ & $0,07438 \mathrm{~b}$ & $0,07729 \mathrm{~b}$ \\
\hline
\end{tabular}

(1) $\mathrm{V}(\mathrm{CR})$ = volume médio da cubagem rigorosa; $\mathrm{V}(f)$ = volume médio estimado em função do fator de forma; $\mathrm{V}(Q)$ = volume médio estimado em função do quociente de forma; $\mathrm{V}($ Mod. 1$)$ = volume médio estimado em função do modelo ajustado de Schumacher e Hall logaritmizado; V(Mod. 2) = volume médio estimado em função do modelo ajustado de Spurr; ${ }^{(2)}$ Médias seguidas pela mesma letra na linha não diferem entre si pelo teste de Duncan a 5\% de significância.

Nos dois regimes de manejo estudados, o volume obtido através do quociente de forma foi estatisticamente superior aos volumes obtidos através dos outros métodos. Rocha et al. (2010), utilizando quociente de forma de Girard (obtenção do diâmetro a 5,2 $\mathrm{m}$ da altura do fuste) também constataram superestimação da variável volume através desse método.

Os volumes estimados a partir do emprego das equações ajustadas e do fator de forma apresentaram valores estatisticamente iguais entre si e em relação aos volumes obtidos na cubagem, o que demonstra a eficiência destes dois métodos na estimação do volume para a espécie, tanto no regime de alto fuste quanto no regime de talhadia. Apesar disso, foi possível notar uma leve tendência do fator de forma a superestimar o volume quando comparado aos valores obtidos por meio das equações ajustadas. Guimarães \& Leite (1996), estudando equações volumétricas para E. grandis, também encontraram volumes estimados similares aos obtidos pela cubagem rigorosa.

\section{Conclusões}

Os modelos de Schumacher e Hall logaritmizado e de Spurr apresentaram melhores performances para estimar o volume de E. urophylla manejado sob regime de alto fuste ou sob regime de talhadia.

Entre os métodos utilizados para estimar o volume, o mais recomendado foi o emprego de modelos volumétricos ajustados e, em seguida, o fator de forma.

$\mathrm{O}$ quociente de forma não apresentou resultados satisfatórios na predição do volume.

\section{Referências}

ANUÁRIO ESTATÍSTICO DA ABRAF 2010: ano base 2009. Brasília, DF: ABRAF, 2010. 140 p.
ALMEIDA, R. C.; CABACINHA, C. D.; ROCHA, T. B.; PAULA, A. Volumetria de Eucalyptus urophylla S. T. Blake pelo método geométrico no Planalto de Conquista, Bahia. Revista Caatinga, Mossoró, v. 24, n. 1, p. 65-75, jan./mar. 2011.

AZEVEDO, T. L.; MELLO, A. A.; FERREIRA, R. A.; SANQUETTA, C. R.; NAKAGIMA, N. Y. Equações hipsométricas e volumétricas para um povoamento de Eucalyptus sp. localizado na FLONA do Ibura, Sergipe. Revista Brasileira de Ciências Agrárias, Recife, v. 6, n. 1, p. 105-112, 2011.

CAMPOS, J. C. C.; TREVIZOL JÚNIOR, T. L.; PAULA NETO, F. Ainda, sobre a seleção de equações de volume. Revista Árvore, Viçosa, MG, v. 9, n. 2, p. 115-126, 1985.

CANTERI, M. G.; ALTHAUS, R. A.; VIRGENS FILHO, J. S.; GIGLIOTI, E. A.; GODOY, C. V. SASM-Agri: sistema para análise e separação de médias em experimentos agrícolas pelos métodos Scoft-Knott, Tukey e Duncan. Revista Brasileira de Agrocomputação, Ponta Grossa, PR, v. 1, n. 2, p. 18-24, 2001.

COUTO, H. T. Z.; BASTOS, N. L. M. Modelos de equações de volume e relações hipsométricas para plantações de Eucalyptus no estado de São Paulo. IPEF, Piracicaba, SP, n. 37. p. 33-44, dez. 1987.

FRANCO, E. J.; SCOLFORO, J. R. S.; MELLO, J. M.; OLIVEIRA, A. D. Eficiência dos métodos para estimativa volumétrica de Eucalyptus camaldulensis. Cerne, Lavras, MG, v. 3, n. 1, p. 82-117, 1997.

FURNIVAL, G. M. An index for comparing equations used in constructing volume tables. Forest Science, Washington, DC, v. 7, n. 4, p. 337-341, 1961.

GUIMARÃES, D. P.; LEITE, H. G. Influência do número de árvores na determinação de equação volumétrica para Eucalyptus grandis. Scientia Forestalis, Piracicaba, SP, n. 50, p. 37-42, 1996.

MACHADO, S. A.; NASCIMENTO, R. G. M.; AUGUSTYNCZIK A. L. D.; SILVA, L. C. R.; FIGURA, M. A.; PEREIRA, E. M.; TÉO, S. J. Comportamento da relação hipsométrica de Araucaria angustifolia no capão da Engenharia Florestal da UFPR. Pesquisa Florestal Brasileira, Colombo, n. 56, p. 5-16, jan./jun. 2008.

McTAGUE, J. P.; BATISTA, J. L. F.; STEINER, L. H. Equações de volume total, volume comercial e forma do tronco para plantações de Eucalyptus no Estado de São Paulo e Rio de Janeiro. IPEF, Piracicaba, SP, n. 41/42, p. 56-63, 1989. 
MIGUEL, E. P.; CANZI, L. F.; RUFINO, R. F.; SANTOS, G. A. Ajuste de modelo volumétrico e desenvolvimento de fator de forma para plantios de Eucalyptus grandis localizados no município de Rio Verde-GO. Enciclopédia Biosfera, Goiânia, v. 6, n. 11, 2010.

\section{MORAES NETO, S. P. Estimativas de volume de Pinus} tecunumanii no Cerrado do Distrito Federal. Planaltina, DF: Embrapa Cerrados, 2009. 16 p. (Embrapa Cerrados. Boletim de pesquisa e desenvolvimento, 230).

NOVAES, A. B. de; LONGUINHOS, M. A. A.; RODRIGUES, J.; SANTOS, I. F. dos; GUSMÃO, J. C. Caracterização e demanda florestal da Região Sudoeste da Bahia. In: SIMPÓSIO SOBRE REFLORESTAMENTO NA REGIÃO SUDOESTE DA BAHIA, 2., 2005, Vitória da Conquista. Memórias. Colombo: Embrapa Florestas, 2008. p. 25-43.

OLIVEIRA, M. L. R.; LEITE, H. G.; GARCIA, S. L. R.; CAMPOS, J. C. C.; SOARES, C. P. B.; SANTANA, R. C. Estimação do volume de árvores de clones de eucalipto pelo método da similaridade de perfis. Revista Árvore, Viçosa, MG, v. 33, n. 1, jan./fev. 2009.

ROCHA, T. B.; CABACINHA, C. D.; ALMEIDA, R. C.; PAULA, A.; SANTOS, R. C. Avaliação de métodos de estimativa de volume para um povoamento de Eucalyptus urophylla S. T. Blake no Planalto da Conquista-BA. Enciclopédia Biosfera, Goiânia, v. 6, n. 10, 2010.

SCHNEIDER, P. R.; COELHO, M. C. B.; ZANON, M. L.; FINGER, C. A. G.; KLEIN, J. E. M. Equações de volume para Eucalyptus dunnii Maiden, determinadas para a depressão central do Estado do Rio Grande do Sul. Ciência Rural, Santa Maria, RS, v. 27, n. 3, p. 425-428, 1997.
SCOLFORO, J. R. Mensuração Florestal 3: relações quantitativas em volume, peso e a relação hipsométrica. Lavras, MG: ESAL/FAEPE, 1993. 292 p.

SILVA, J. A. A.; BAILEY, R. L.; MEUNIER, I. M. J. Método das quatro árvores para estimativas volumétricas em plantios florestais. Boletim de Pesquisa Florestal, Colombo, n. 8/9, p. 64-91, jun./dez. 1984.

SILVA, J. N. M.; ARAÚJO, S. M. Equação de volume para árvores de pequeno diâmetro na Floresta Nacional dos Tapajós. Boletim de Pesquisa Florestal, Colombo, n. 8/9, p. 16-25, jun./ dez. 1984.

SOARES, C. P. B.; NETO, F. P.; SOUZA, A. L. Dendrometria e inventário florestal. Viçosa, MG: Universidade Federal de Viçosa, 2006. $276 \mathrm{p}$.

SOARES, C. P. B.; SILVA, G. F.; MARTINS, F. B. Influence of section lengths on volume determination in eucalyptus trees. Cerne, Lavras, MG, v. 16, n. 2, p. 155-162, abr./jun. 2010.

THIERSCH, A. A eficiência das distribuições diamétricas para prognose de Eucalyptus camaldulensis. 1997. 155 f. Dissertação (Mestrados em Engenharia Florestal) - Universidade Federal de Lavras, Lavras, MG.

THOMAS, C.; ANDRADE, C. M.; SCHNEIDER, P. R.; FINGER, C. A. J. Comparação de equações volumétricas ajustadas com dados de cubagem e análise de tronco. Ciência Florestal, Santa Maria, RS, v. 16, n. 3, p. 319-327, 2006.

TONINI, H.; COSTA, M. C. G.; SCHWEENGBER, L. A. M. Crescimento da teca (Tectona grandis) em reflorestamento na Amazônia Setentrional. Pesquisa Florestal Brasileira, Colombo, n. 59, p. 5-14, jul./dez. 2009. doi: 10.4336/2009.pfb.59.05 